\title{
Deutsche Gesellschaft für Hirnstimulation in der Psychiatrie
}

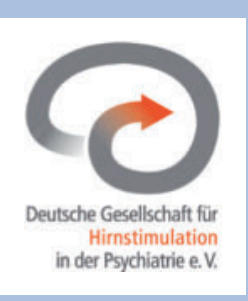

\section{Jahrestagung der Deutschen Gesellschaft für Hirnstimulation in der Psychiatrie}

Ein großer Erfolg war die 10. Jahrestagung der Deutschen Gesellschaft für Hirnstimulation in der Psychiatrie e. V., die 2019 von der Gesellschaft in Zusammenarbeit mit Prof. Landgrebe, kbo-Lech-Mangfall-Klinikum in Agatharied ausgerichtet wurde. Zu den Fokusthemen zählten Standards der TMS-Behandlung bei depressiven Störungen und Schizophrenie sowie die transkranielle Gleichstromstimulation. Ein eigener Track widmete sich den Besonderheiten der Hirnstimulationsbehandlung in der Gerontopsychiatrie, im Kindes- und Jugendalter sowie in Schwangerschaft und Stillzeit. Prof. Hajak gab einen Überblick über 20 Jahre TMS in der Psychiatrie - gestern, heute und morgen. Während der Tagung traf sich zudem das German Center for Brain Stimulation (GCBS, Leitung Prof. Padberg, München). Auf der DGPPN-Tagung 2019 beschloss der Vorstand das Programm für die Jahrestagung 2020, die diesmal von Dr. Heiko UIIrich am 18. Und 19. Juni 2020 in Siegen

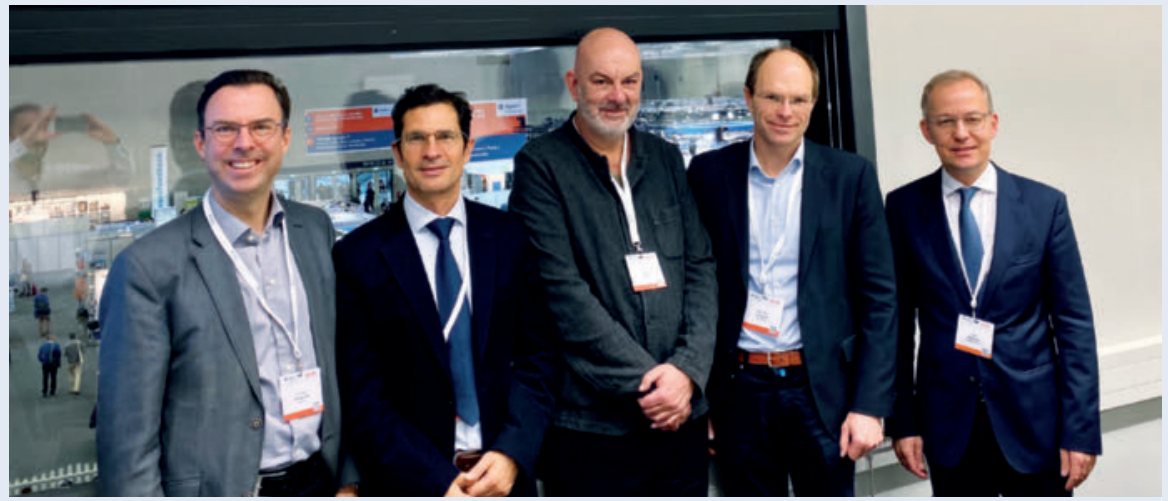

- Abb. 1 Vorstandsmitglieder der DGHP 2019. Von links: Michael Landgrebe, Carlos Schönfeldt-Lecuona, Heiko Ullrich, Bertold Langguth, Peter Zwanzger (Quelle: Peter Zwanzger).

durchgeführt wird. Neben zahlreichen Aspekten der Anwendungsmöglichkeiten in Neurologie und Psychiatrie werden dieses Jahr auch ethische Aspekte im Vordergrund stehen. Ein Weiterbildungsworkshop zur Anwendung von TMS in der Praxis wird ebenfalls stattfinden.

\section{IMPRESSUM}

Prof. Dr. Carlos Schönfeldt-Lecuona Schriftführer

Deutsche Gesellschaft für Hirnstimulation in der Psychiatrie e.V.

Psychiatrische Univ.-Klinik Ulm Leimgrubenweg 12, 89075 Ulm www.dghp-online.de info@dghp-online.de 